\title{
The Level of utilization and Satisfaction of Complete Denture Treatment Provided in Fiji from 2010-2016
}

\author{
Kartika Kajal $^{1}$, Masoud Mohammadnezhad ${ }^{2}$, Gowri Sivaramakrishnan ${ }^{3}$, Kritesh Bhai ${ }^{4}$ \& Sabiha Khan ${ }^{2}$ \\ ${ }^{1}$ School of Dentistry and Oral Health, Fiji National University, Fiji \\ ${ }^{2}$ School of Public Health and Primary Health Care, Fiji National University, Fiji \\ ${ }^{3}$ Ministry of Health, Kingdom of Bahrain, Bahrain \\ ${ }^{4}$ BDS (Fiji), Post Graduate Certificate in Tertiary Teaching (Fiji), Fiji \\ Correspondence: Dr Masoud Mohammadnezhad, School of Public Health \& Primary Care, Fiji National \\ University, Fiji. Tel: 679-331-1700. E-mail: masoud.m@fnu.ac.fj
}

\author{
Received: October 27, 2021 Accepted: November 26, $2021 \quad$ Online Published: December 6, 2021 \\ doi:10.5539/gjhs.v14n1p43 URL: https://doi.org/10.5539/gjhs.v14n1p43
}

\begin{abstract}
Background: Wearing dentures helps rehabilitate the oral health status for the older people. Due to lack of previous data, this objective of this study was to determine the level of utilization and satisfaction of complete denture treatment at dental clinics in Fiji.

Materials and Methods: A cross sectional descriptive study was conducted on 85 edentulous patients and dentures at the Fiji National University (FNU) teaching dental clinic from 2010-2016.Patients who utilized the dentures fabricated at FNU and identified as complete denture patients from 2010-2016 were included in this study. Data was collected using the denture assessment form and Oral Health Impact Profile for Edentulous Patients (OHIP-EDENT) questionnaire. Correlation test was done and p less than 0.05 was considered as level of significance.
\end{abstract}

Results: Participants were between the age range of 40-79 years with majority being male (54\%). Greater level of satisfaction noted among 60-69 years male participants and those wearing the dentures for 3-4 years than other groups. Difficulty in chewing (40\%) and food catching underneath the denture $(33 \%)$ were common denture related complains. One thirds of participants reported of uncomfortable dentures while $24 \%$ had sore spots due to their dentures. More than two thirds of them, $(71 \%)$ were unable to eat and enjoy food and for most patients, the vertical dimension (94\%), esthetics assessment (94\%), tooth selection (91\%), boarder extension (78\%), posterior seal (99\%) and centric relation (99\%) were acceptable. Patients with acceptable vertical dimension (46.1 \pm 7.6$)$, acceptable esthetics $(45.8 \pm 7.9)$ and boarder extension (48.0 \pm 7.2$)$ showed greater satisfaction level compared to those with unacceptable denture characteristics.

Conclusion: This study highlighted that satisfaction among recent denture wearers was low and majority participants portrayed poor understanding regarding denture aftercare and hygiene. It can be recommended for the teaching institutes and dental clinics to evaluate the quality of treatments and help to improve the quality of services being provided to the patients. Further study is needed to determine the in- depth perceptions of denture wearers. It also can be recommended for the patients to take heed of the post operative denture hygiene instructions being given.

Keywords: Complete denture, Denture assessment, Edentulism, Fiji, Patient's satisfaction

\section{Introduction}

Dental caries, edentulism, periodontal disease and oral cancer are currently amongst some of the major public health issues around the globe (Meier et al., 2017) with significant differences between countries (Emami, de Souza, Kabawat and Feine, 2013). Edentulism has been defined as the loss of all-natural dentition (Ishtiaq Ali et al., 2019; Tyrovolas et al., 2016; Pengpid \& Peltzer, 2018). Edentulism remains a major disease burden in older adults' worldwide (Ishtiaq Ali et al., 2019). The World Health Organisation (WHO) states the incidence of edentulism is about 7\%-69\% internationally (Ishtiaq Ali et al., 2019). Apart from elderly, edentulism also affects young age groups being more prevalent in the socially deprived groups (Tyrovolas et al., 2016). Edentulism diminishes the 
quality of life of individuals as it affects an individual's oral and general health (El-Dahdah, 2016; Jain, Kaushik, Dhawan, \& Tandan, 2018; Michaud, de Grandmont, Feine, \& Emami, 2012; Pengpid \& Peltzer, 2018; Deniz \& Kulak Ozkan, 2012). tooth loss often limits a person's ability to eat and speak well, even to the extent of limiting a person's ability to participate in social activities thereby affecting an individual's overall quality of life (Khan \& Khan, 2015; Deniz \& Kulak Ozkan, 2012). Edentulous individuals are also in greater risk for different systemic diseases and an increase in mortality rate (Emami et al., 2013). Although complete dentures (false teeth) cannot be considered as a substitute or natural dentition, they remain to be a staple treatment option for edentulous patients (Emami, de Souza, Kabawat, \& Feine, 2013).

The number of patients who need conventional complete denturers is on a rise (Negahdari et al., 2016) and is the most common type of prosthesis used (Reginato et al., 2017; G Ashok \& Ganapathy, 2017). The demand for dentures (or other prosthetic rehabilitation) is increasing dramatically due to an increase in the rate of edentulousness (Khan \& Khan, 2015; Srisilapananet al., 2016; Deniz \& Kulak Ozkan, 2012; Santos et al., 2015). Hence, Esan et al., (2004) stated that the demand for dentures was higher in participants of lower level of education and low socio-economic status. Many edentulous patients benefit from complete denture treatment and have reported satisfactory oral and masticatory function with their use (Carlsson \& Omar, 2010). Wearing dentures helps rehabilitate the oral health status for the older people. It improves the chewing ability and makes oral health function better. Hence, this improves the quality of life (Srisilapanan et al., 2016). However, even when the dentures satisfy all the prosthodontic criteria, not all complete denture wearers are able to adapt to their dentures (Carlsson \& Omar, 2010). Around 65\%- 90\% of the individuals are generally satisfied with their dentures (Carlsson \& Omar, 2010). Complains with removable dental prosthesis are associated with a lot of factors, such as retention, stability, dimensios, occlusion, esthetics, speech, difficulty in chewing, food accumulation, (G Ashok and Ganapathy, 2017; Negahdari et al., 2016; El-Dahdah, 2016) thick or over extended denture flange, tight or loose dentures, occlusal interference, frenal impingements and so forth. These problems can arise due to alteration of anatomical structures, difficulty in adaptation or some technical faults in denture design and fabrication (G Ashok \& Ganapathy, 2017). Unsatisfactory dentures may cause issues such as alteration in taste and smell, masticatory issues, oral discomfort, oral ulcerations, denture stomatitis and so forth (El-Dahdah, 2016).

There have been very few studies published in Fiji with regards to the use of prosthesis. King and Kapadia (2003) reported around $63 \%$ of edentulous patients in Fiji had no dentures while the other $37 \%$ had full upper and lower dentures. In their study, King and Kapadia (2003) stated about $41 \%$ patients required adjustment with their dentures as they had some denture related issues.

Prosthodontic complete denture treatment has been into practice at the School of Dentistry and Oral Health (SDOH), Fiji National University (FNU) from the very beginning of the dental school. However, the outcome of these dentures fabricated by the students in terms of utilization and satisfaction over the years had never been yet evaluated. This understanding greatly helps in improving the quality of treatment provided. Hence, the objective of the study was to understand the level of utilization and satisfaction of complete denture treatment provided at FNU dental clinics from 2010-2016.

\section{Materials and Methods}

\subsection{Study Design and Setting}

This was a cross- sectional descriptive study on complete denture patients selected from years 2010-2016 and their level of satisfaction and oral health related quality of life. This study was conducted at FNU dental clinics in Suva, Fiji. FNU dental clinics are the only teaching institute in Fiji that offers dental courses.

\subsection{Study Population and Sample}

The study population comprised of all the edentulous patients who received removable complete (full upper and lower) dentures at FNU dental clinics. The study participants were selected after analyzing files detailing treatments performed from 2010-2016, which were kept at the FNU dental clinics. Selection was done based on the inclusion and exclusion criteria. The inclusion criteria comprised of; student fabricated dentures made at FNU dental clinics, complete denture patients only from 2010-2016, patients should be utilizing those dentures fabricated at FNU, and male and female patients of any age. The exclusion criteria on the contrary included; those patients with serious systemic conditions who could not avail themselves for the interview.85 patients were interviewed and dentures were examined.

\subsection{Data Collection Tools}

The tools used for data collection were a modified version of the Oral Health Impact Profile for Edentulous Patients (OHIP-EDENT) questionnaire to gauge the patient satisfaction which were developed after literature 
search (Reginato et al., 2017; Michaud et al., 2012) and a self-developed Oral Examination and Denture Assessment (OEDA) form.

\subsubsection{OHIP-EDENT Questionnaire}

The OHIP-EDENT questionnaire consisted of seven sections. They include Functional Limitation which had 3 questions, Physical Pain with four related questions, two questions under Psychological discomfort, three questions under Physical disability, two questions under Psychological disability, three questions under Social disability and two questions under the sub-heading Handicap.

Each question in OHIP-EDENT had options of 'yes', 'no' and 'sometimes'. Scores were given for each for the purpose of analysis; 'yes' denoted a score of 1, 'sometimes' was denoted a score of 2 while 'no' had a score of 3. This helped in determining the patient satisfaction levels.

Both data collection tools were pretested prior to its administration. The content of the tools was checked by 3 experts and they were also shown to 5 participants to make sure they are understandable by the study participants.

In terms of the variables, the independent variables included; age, gender, location, occupation while the dependent variable were patient satisfaction and utilization of their dentures, and the years of denture use.

\subsubsection{OEDA Form}

The OEDA form had two sections. The first section under oral examination and denture assessment was based on the denture history (patients' perception of his/her dentures) while the second section focused on denture assessment (dentists' perception of the denture).

\subsection{Study Procedure}

Complete denture patients were selected based on the set inclusion criteria by the principal investigator. Every participant was given a written form of information sheet as well as a verbal explanation on the purpose of this study. The information sheet was provided in three different laguages; English, Hindi and Itaukei. Approval was requested from the participants in the form of a written consent. Moreover, every participant was given an identification code instead of using their actual names; to maintain confidentiality. The patients were examined for initial denture assessment using the OEDA form following which the OHIP-EDENT questionnaire was administered by the principal investigator.

\subsection{Data Management and Analysis}

Data was collected and descriptive statistical analysis was done inSPSS. Significance was set at $\mathrm{p}<0.05$.

\subsection{Ethical Approvals}

Ethical approvalswere obtained from the Department of Oral Health Research Committee (DOHRC) and also form the College Health Research Ethics Committee (CHREC) at FNU.

\section{Results}

A total of 85 participants with full upper and lower set of dentures were examined. Majority (56\%) participant were between the age of 60-69 while only 5\% participants were between the age range of 40-49. About half of the participants $(46 \%)$ were male and $54 \%$ were female. Participants had at least 1 to more than 5 years of experience of wearing the dentures (Table 1). 
Table 1. Demographic characteristics of participants

\begin{tabular}{ll}
\hline Characteristics & Frequency (\%) \\
\hline Age & \\
$40-49$ & $4(5)$ \\
$50-59$ & $17(20)$ \\
$60-69$ & $48(56)$ \\
$70-79$ & $16(19)$ \\
\hline Gender & \\
Male & $46(54)$ \\
Female & $39(46)$ \\
\hline Denture wearing experience & \\
$1-2$ & $33(39)$ \\
$3-4$ & $27(32)$ \\
$5+$ & $25(29)$ \\
\hline
\end{tabular}

Participants between 60-69 years were more satisfied with their denture $(46.1 \pm 8.3)$ while participants between 70-79 were least satisfied (43.3 \pm 7.5$)$. Male participants showed more satisfaction (46.5 \pm 7.6$)$ than female participants (44.2 \pm 8.6$)$. Participants wearing the dentures for 3-4 years had greater satisfaction (46.4 \pm 6.5$)$ while those who recently received their dentures had least satisfaction (44.2 \pm 8.8 ) (Table 2). There was no statistical significance difference between these variables and level of satisfaction.

Table 2. Correlation between independent variables and satisfaction level

\begin{tabular}{llc}
\hline Variables & Satisfaction & p- value \\
\hline Age & & \\
$40-49$ & $45.5 \pm 9.3$ & 0.671 \\
$50-59$ & $44.6 \pm 8.4$ & 0.209 \\
$60-69$ & $46.1 \pm 8.3$ & \\
$70-79$ & $43.3 \pm 7.5$ & \\
\hline Gender & & 0.575 \\
Male & $46.5 \pm 7.6$ & \\
Female & $44.2 \pm 8.6$ & \\
\hline Years of denture wearing & & \\
$1-2$ & $44.2 \pm 8.8$ & \\
$3-4$ & $46.4 \pm 6.5$ & \\
$5+$ & $45.5 \pm 9.0$ & \\
\hline
\end{tabular}

Majority of participants (94\%) felt their dentures were very important to them, $5 \%$ felt it was less important while $1 \%$ felt it was not important at all. Moreover, $93 \%$ participants reported of receiving adequate information during the denture making process while the rest $(7 \%)$ were not satisfied with the amount and the type of information they received. Participants who received adequate information showed greater satisfactions levels (45.8 \pm 8.1$)$ than the rest (38.2 \pm 5.7$)$.

Common denture related complains given by participants related to functional limitations were difficulty in chewing (40\%) and food catching underneath the denture (33\%). These functional limitations brought about psychological discomfort in participants and caused physical and social disability.34\% participants reported of uncomfortable dentures while $24 \%$ had sore spots due to their dentures. Participants had become more self-conscious (19\%) and only 5\% had become embarrassed because of their dentures. More than two thirds of them, $(71 \%)$ were unable to eat and enjoy food and $33 \%$ got interrupted while eating meals. A few participants had social disability whereby only $6 \%$ avoided socializing, $1 \%$ had become less tolerant of the spouse and only $1 \%$ 
became more irritable of other people. More than half of them (53\%) were unable to enjoy others company and only $1 \%$ stated that life had become less satisfyingwhile $93 \%$ participants reported their overall quality of life had improved after getting dentures (life was satisfying) (Table 3).

Table 3. Participant satisfaction, utilization and denture related complains $(\mathrm{n}=85)$

\begin{tabular}{lccc}
\hline \multirow{2}{*}{ Variables } & \multicolumn{3}{c}{ Frequency (\%) } \\
\cline { 2 - 4 } Functional limitation: & Yes & Sometimes \\
Difficulty chewing & $34(40)$ & $51(60)$ & $0(0)$ \\
Food catching underneath the denture & $28(33)$ & $31(36)$ & $26(31)$ \\
Denture not fitting & $35(41)$ & $50(59)$ & $0(0)$ \\
\hline Physical Pain: & & & \\
Painful aching & $25(29)$ & $60(71)$ & $0(0)$ \\
Sore spots & $20(24)$ & $65(76)$ & $0(0)$ \\
Uncomfortable dentures & $29(34)$ & $56(66)$ & $0(0)$ \\
\hline Psychological discomfort/ disability: & & & \\
Worried because of denture issues & $11(13)$ & $64(75)$ & $10(12)$ \\
Become more self-conscious & $16(19)$ & $62(73)$ & $7(8)$ \\
Upset because of denture issues & $5(6)$ & $60(70)$ & $20(24)$ \\
Embarrassed & $4(5)$ & $61(71)$ & $20(24)$ \\
\hline Physical disability: & & & \\
Avoid eating certain food & $46(54)$ & $36(42)$ & $3(4)$ \\
Able to eat and enjoy food & $60(71)$ & $25(29)$ & $0(0)$ \\
Get interrupted while eating meals & $28(33)$ & $39(46)$ & $18(21)$ \\
\hline Social disability: & & & \\
Avoid socializing & $5(6)$ & $66(78)$ & $14(16)$ \\
Have become less tolerant of the spouse & $1(1)$ & $80(94)$ & $4(5)$ \\
Have become irritable of people & $1(1)$ & $80(94)$ & $4(5)$ \\
\hline Handicap: & & & $8(9)$ \\
Unable to enjoy others company & $45(53)$ & $32(38)$ & $5(6)$ \\
Life has become less satisfying & $1(1)$ & $79(93)$ & \\
\hline & & & \\
\hline
\end{tabular}

Patients also had issues with denture retention. One third of them (34\%) reported their denture falls when in function with $66 \%$ having no retention issues. $28 \%$ reported both upper and lower denture had become loose overtime and not fitting well, $11 \%$ complained of only lower denture being loose, $2 \%$ stated the upper denture was loose only while $59 \%$ had no issues as such. Moreover, denture esthetics is an important issue often resulting in patient dissatisfaction. Participants complained about the size and color of teeth selected and comparing current dentures to either their old dentures or natural dentition. Dentist's perception of esthetic assessment stated that $94 \%$ had acceptable esthetics while the rest did not. Among those with unacceptable esthetics included patients who had protruded facial profile, unacceptable smile line, greater discrepancies felt between the upper and lower facial profile or teeth being covered with acrylic and not giving a pleasant appearance. $91 \%$ of the patients has acceptable mold of teeth selected, while $9 \%$ had either very large or very small teeth selected not suiting the facial profile, teeth were either worn out or excessively grinded to get correct occlusion.

The dentist examined the patient dentures using the denture assessment form. For most patients, the vertical dimension (94\%), esthetics assessment (94\%), tooth selection (91\%), boarder extension (78\%), posterior seal (99\%) and centric relation (99\%) were acceptable.

The denture characteristics were correlated to patient satisfaction levels. Patients with acceptable vertical

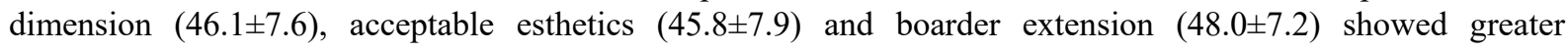


satisfaction level compared to those with unacceptable denture characteristics (Table 4).

Table 4. Correlation between acceptable characteristics of complete denture andpatient satisfaction

\begin{tabular}{lcc}
\hline Variables & Patient satisfaction & p- value \\
\hline Vertical dimension & & $<0.0001^{*}$ \\
Acceptable & $46.1 \pm 7.6$ & \\
Not Acceptable & $31.8 \pm 2.0$ & $0.018^{*}$ \\
\hline Esthetic Assessment & & \\
Acceptable & $45.8 \pm 7.9$ & \\
Not Acceptable & $36.6 \pm 7.8$ & 0.062 \\
\hline Tooth selection & & \\
Acceptable & $45.8 \pm 8.0$ & $<0.0001^{*}$ \\
Not Acceptable & $40.1 \pm 8.2$ & \\
\hline Border Extension & & \\
Acceptable & $48.0 \pm 7.2$ & 0.400 \\
Not Acceptable & $37.0 \pm 4.8$ & \\
\hline Posterior Seal & & \\
Acceptable & $45.4 \pm 8.1$ & \\
Not Acceptable & $36.0 \pm \mathrm{SD}$ & \\
\hline Centric Relation & & \\
Acceptable & $33.0 \pm \mathrm{SD}$ & \\
Not Acceptable & & \\
\hline
\end{tabular}

Participants generally had poor understanding about denture aftercare and hygiene. Most participants used toothpaste to clean their dentures (81\%). A few participants also slept with their dentures $(20 \%)$ as well while the rest took out their dentures and placed them in water before sleeping $(80 \%)$. Other aids which the participants used to clean their dentures were; using bleaching agent (5\%), $6 \%$ used denture cleaning tablets while $12 \%$ used soap and water to clean their dentures. Only $2 \%$ participants reported of cleaning the denture every time after they ate (Table 5).

Table 5. Participant's behavior related to Denture after-care and hygiene

\begin{tabular}{lcc}
\hline \multirow{2}{*}{ Participant comments } & \multicolumn{2}{c}{ Frequency (\%) } \\
\cline { 2 - 3 } & $69(81)$ & No \\
\hline Uses toothpaste to wash & $68(80)$ & $16(19)$ \\
Takes denture out at night before sleeping & $17(20)$ & $68(80)$ \\
Sleeps with denture & $4(5)$ & $81(95)$ \\
Washes with bleaching agent & $5(6)$ & $80(94)$ \\
Uses tablets to clean & $10(12)$ & $75(88)$ \\
Uses soap and water to wash denture & $2(2)$ & $83(98)$ \\
Cleans denture after eating as well & & \\
\hline
\end{tabular}

\section{Discussion}

The objective was to determine the level of utilization and satisfaction of complete denture treatment at dental clinics in Fiji. This study was conducted among edentulous participants between 40-79 years of age. Majority (56\%) participant were between the age of 60-69 while only 5\% participants were between the age range of 40-49. About half of the participants (46\%) were male and $54 \%$ were female. 


\subsection{Patient Satisfaction with Dentures in Relation to Demographic Characteristics}

In this study, participants between 60-69 years were more satisfied with their denture (46.1 \pm 8.3$)$ while participants

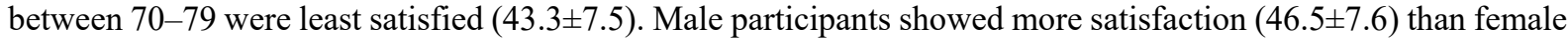
participants (44.2 \pm 8.6 ). Participants wearing the dentures for 3-4 years had greater satisfaction (46.4 \pm 6.5$)$ while those who recently received their dentures had least satisfaction (44.2 \pm 8.8 ). Patients with two complete dentures have longer prosthetic history and are more experienced in the adaptation process than removable partial denture wearers (Komagamine et al., 2016). Thus, shortening the time necessary for adaptation to complete denture wearers (Komagamine et al., 2016). Komagamine et al., (2016) showed a relationship between sex and adaptability to dentures; men were reported to adapt more rapidly to new dentures than women. Similarly, there are other possible factors that might determine the prognosis of complete denture treatment. For instance; previous denture experience, the period of existing denture use, numbers of previous complete dentures, and duration of edentulousness (Komagamine et al., 2016).

\subsection{Denture Related Complains and Functional Limitation}

Common denture related complains given by participants related to functional limitations were difficulty in chewing (40\%) and food catching underneath the denture (33\%). These functional limitations brought about psychological discomfort in participants and caused physical and social disability. 34\% participants reported of uncomfortable dentures while $24 \%$ had sore spots due to their dentures. Participants had become more self-conscious (19\%) and only $5 \%$ had become embarrassed because of their dentures. More than two thirds of them, $(71 \%)$ were unable to eat and enjoy food and $33 \%$ got interrupted while eating meals. A few participants had social disability whereby only $6 \%$ avoided socializing, $1 \%$ had become less tolerant of the spouse and only $1 \%$ became more irritable of other people. More than half of them (53\%) were unable to enjoy others company and only $1 \%$ stated that life had become less satisfying. Participants in this study had issues with mastication which were brought about because of pain along the edentulous ridges due to presence of undercuts and sore spots because of unpolished dentures or overextensions. Patients that reported of problems with speech were majority recent denture wearers or those that had existing dentures thus having problems with adaptations. Such patients had issues with pronunciation of words and complained of difficulty during telephone conversations. Food accumulation underneath dentures was also reported in this study. Khan and Khan (2015) stated common complaints reported by complete denture wearers include; difficulty in speech, mastication, pain and esthetic issues. Pain may often cause insomnia and some negative changes in eating behaviours. These problems are often associated with the elderly. The most common areas of dissatisfaction are as follows: mastication, overall perception, speech, denture cleanliness, fit, natural tooth problems, appearance and odour (Khan \& Khan, 2015). Pain was reported as one of the most common dentures related complains (Parvez et al., 2020 apart from loose denture, mastication and speech (Salih et al., 2016). Speech issues was noted higher within the age group of up to 60 years 11 (5.5\%) (Salih et al., 2016). Speech was not an issue in a study conducted by Bhat et al., (2014) as majority (58\%) participants were satisfied with it. Only 3\% participants had issues with mastication while the rest were satisfied (Bhat et al., 2014). Very few participants had issues with denture retention (Bhat et al., 2014). Mastication discomfort and looseness of denture were the most common complaints followed by lack of retention, bad breath, tongue restriction and food accumulation (Sanyal et al., 2013). Torres-Sanchez et al., (2018) reported of better retention stability and less accumulation of particles of the food particles underneath the denture with the use of denture adhesives. Perea et al., (2013) reported that the most affected domains were "functional limitation" and "physical pain", followed by "physical disability". Minor impacts were recorded for the psychological and social subscales ("psychological discomfort", "psychological disability", "social disability" and "handicap") (Perea et al., 2013). Women complained about the appearance of their dentures, while more men had objections regarding mastication (Komagamine et al., 2016; Salih et al., 2016).

One third of them (34\%) reported their denture falls when in function with $66 \%$ having no retention issues. $28 \%$ reported both upper and lower denture had become loose overtime and not fitting well, 11\% complained of only lower denture being loose, $2 \%$ stated the upper denture was loose only while $59 \%$ had no issues as such. Zani et al (2009), reported that patients often express dissatisfaction with their lower arch dentures. Frequent complaints with regards to this include; reduced retention and stability of conventional dentures, difficulties with mastication and verbal communication, which are often due to resorption of alveolar bone.

\subsection{Denture Assessment and Satisfaction Levels}

The denture characteristics were correlated to patient satisfaction levels. Patients with acceptable vertical

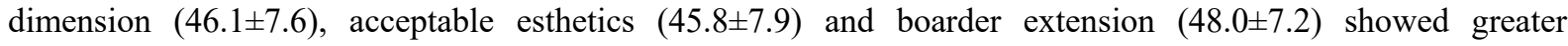
satisfaction level compared to those with unacceptable denture characteristics. Majority participants from this 
study, who were assessed by the dentist, had acceptable vertical dimension (94\%), esthetics assessment (94\%), tooth selection $(91 \%)$, boarder extension $(78 \%)$, posterior seal $(99 \%)$ and centric relation $(99 \%)$. Patients who reported of looseness of lower dentures mostly had resorbed edentulous ridges. $6 \%$ patients had unacceptable esthetic (according to the dentist that inspected the participant dentures) which was due to wrong type of tooth selection (color and size). Esthetic issues were mostly seen in female participants than to male counterparts with regards to the size, shape and color of teeth selected. Patients had higher speech and esthetics satisfaction levels, but greater mastication complaints with regards to their existing dentures (Komagamine et al., 2016) with similar results obtained from this study. This suggests that in the mastication, speech, and esthetics recovery from mastication problems is most difficult. In complete denture therapy there is a need to cover the palate and the thickness of the cover might cause a prolonged adaptation period (Komagamine et al., 2016). In a study by Bhat et al., (2014), majority participants were either happy or very happy with the denture esthetics in terms of denture colour $(88 \%)$, colour of the teeth in the denture $(93 \%)$, overall facial appearance $(93 \%)$ and denture smoothness (93\%). Khan and Khan (2015) argued that elderly edentulous participants had improved overall oral health related quality of life 16 months post denture insertion, whereby females had reported better quality of life than their male counterparts. A study (Srisilapanan et al., 2016) done in Thai population showed that good quality dentures greatly improve their oral health related quality of life, almost to the level of those with natural dentition which is also reflected in this study. In a study by Bhat et al., (2014), 93\% participants portrayed that they were satisfied with their dentures. However, a study by Parvez et al., (2020) showed low quality of life of complete denture wearers as they frequently had problems in eating, social interaction and communication. Edentulous elderly people who are well satisfied with their daily lives are also satisfied with their complete dentures (Yoshida et al., 2001). Quality of dentures had been reported as the most significant factor in determining patient satisfaction (Celebic et al., 2003). Apart from that, level of education, self-perception of affective and economic status, and quality of life are all related to patient satisfaction (Celebic et al., 2003). The use of conventional complete dentures brings negative impacts in the oral health related quality of life of patients as reported by Perea et al., (2013). Panchevska et al., (2018) reported that patients' satisfaction was not directly related with the quality of complete dentures.

\subsection{Denture Aftercare and Hygiene}

Participants in this study portrayed poor understanding regarding denture aftercare and hygiene. $81 \%$ participants reported of using toothpaste to wash their denture and $20 \%$ slept with the denture overnight. Hence, dentist patient communication is of utmost importance. $93 \%$ participants of this study reported of receiving enough information regarding the denture making process while the rest did not. Most of the individuals used only water to clean their dentures twice per day while others used water and toothbrush for denture cleansing (R, Robin* \& Raj, 2015). Those who had been wearing dentures for longer had maintained better frequency of denture cleaning (R, Robin and Raj, 2015). Poor denture hygiene was reported as one factor that obviously contributed to the occurrence of various denture related complaints such as pain and discomfort (Budală et al., 2021). 29.3\% participants slept with their prosthesis in mouth (Mushtaq et al., 2019). 57.3\% told that they were given only verbal instructions about methods of cleaning while $42.7 \%$ were not even told or shown any method of cleaning of denture by dentist (Mushtaq et al., 2019). Developing dentist patient relationship involves providing the patient with sufficient information at the treatment planning stage and throughout the course (Chen et al., 2015). The patient should be well informed about the denture fabrication process, denture wearing episodes and after care concerns, individual follow up schedules and instructions related to nutrition, speech, nocturnal wear and denture hygiene (Chen et al., 2015). An improvement in quality of complete denture can be brought about by development of effective clinical skills and technical procedures to improve denture occlusion, articulation, maxillary and mandibular retention and stability (Chen et al., 2015). Clinicians should be well aware of the patient expectations and understand the importance of providing good quality dentures (Sanchana, 2015).

\subsection{Limitation}

This study applied a self- reported questionnaire to collect data so the results is this study is heavily depending on participant response. This study also used a small sample size that may affect the power of statistics used. Furthermore, searching for folders to call the patients to the clinic for the research was a bit challenging and time consuming as all the folders were mixed in the archive in the record room.

\section{Conclusion}

Participants between 60-69 years were more satisfied with their denture while participants between 70-79 were least satisfied. Participants wearing the dentures for 3-4 years had greater satisfaction than those who recently received their dentures. Common denture related complains given by participants related to functional limitations were difficulty in chewing and food catching underneath the denture. Other findings reported were uncomfortable 
dentures, sore spots due to their dentures, unable to eat and enjoy food because of uncomfortable dentures and getting interrupted while eating meals. A third of the participants reported issues with denture retention. Patients with acceptable vertical dimension, acceptable esthetics and boarder extension showed greater satisfaction level compared to those with unacceptable denture characteristics. Majority participants portrayed poor understanding regarding denture aftercare and hygiene. Further, it can be recommended for the teaching institutes and dental clinics to evaluate the quality of treatments being provided by the students as this can help improve the quality of services being provided to the patients. A qualitative study can be conducted by future researchers to determine the in- depth perceptions of denture wearers. It can be recommended for the patients to take heed of the post operative denture hygiene instructions being given.

\section{Competing Interests Statement}

The authors declare that there are no competing or potential conflicts of interest.

\section{References}

Abdulazeez, J., Salih, H., \& Aljamoor, C. (2016). Clinical Evaluation of Post Insertion Problems among Newly Wearers of Complete Denture Patients in Sulaimani City. Sulaimani Dental Journal, 3(2), 98-102. https://doi.org/10.17656/sdj.10064

Bhat, V. S., \& Malli, P. (2014). A survey to assess patient satisfaction after receiving complete denture prostheses in AB Shetty Memorial Institute of Dental Sciences. Journal of Health and Allied Sciences NU, 4(02), 081-085. https://doi.org/10.1055/s-0040-1703768

Budală, D. G., Baciu, E. R., Virvescu, D. I., Armencia, A., Scutariu, M. M., Surlari, Z., \& Balcoș, C. (2021). Quality of Life of Complete Denture Wearers-A Comparative Study between Conventional Dentures and $\begin{array}{llllll}\text { Acrylic Dentures with Vitamin B12 Incorporated. Medicina, } & \text { 57(8), } 820 .\end{array}$ https://doi.org/10.3390/medicina57080820

Carlsson, G. E., \& Omar, R. (2010). The future of complete dentures in oral rehabilitation. A critical review. Journal of Oral Rehabilitation, 37(2), 143-156. https://doi.org/10.1111/j.1365-2842.2009.02039.x

Čelebić, A., Knezović-Zlatarić, D., Papić, M., Carek, V., Baučić, I., \& Stipetić, J. (2003). Factors related to patient satisfaction with complete denture therapy. The Journals of Gerontology Series A: Biological Sciences and Medical Sciences, 58(10), M948-M953. https://doi.org/10.1093/gerona/58.10.M948

El-Dahdah, B. P. (2016). Reliability and Validity of Patient's Denture Satisfaction Assessment (PDSA) Questionnaire for Patient-Based OHRQoL Assessment of Complete Dentures (Doctoral dissertation, The University of Texas School of Dentistry at Houston). Retrieved November 12, 2020, from https://www.semanticscholar.org/paper/Reliability-and-Validity-of-Patient\%27s-Denture-for-El-Dahdah/f4f 9e4b5ae373d554088e 5ceaeab3c0fd6943bf7

Emami, E., de Souza, R. F., Kabawat, M., \& Feine, J. S. (2013). The impact of edentulism on oral and general health. International journal of dentistry, 2013. https://doi.org/10.1155/2013/498305

Esan, T. A., Olusile, A. O., Akeredolu, P. A., \& Esan, A. O. (2004). Socio-demographic factors and edentulism: the Nigerian experience. BMC Oral health, 4(1), 1-6. https://doi.org/10.1186/1472-6831-4-3

Ashok, N. G., \& Ganapathy, D. (2017). Evaluation of post-operative complaints in complete denture and removable partial denture wearers: A questionnaire based study. Journal of Pharmaceutical Sciences and Research, 9(9), 1438.

Ali, H. I., Memon, M. R., Shaikh, A. G., Memon, H., \& Samejo, I. (2019). Edentulism in relation to sociodemographic status of patients. Pakistan Oral \& Dental Journal, 39(1), 98-101. Retrieved 12 November, 2020, from https://podj.com.pk/index.php/podj/article/view/359

Jabeen, B., Samejo, I., Hasan, S. M. U., Khan, A., \& Ilyas, Y. (2018). POST INSERTION COMPLAINTS ASSOCIATED WITH NEW COMPLETE DENTURE. Pakistan Oral \& Dental Journal, 38(1), 127-129.

Kaushik, K., Dhawan, P., Tandan, P., \& Jain, M. (2018). Oral health-related quality of life among patients after complete denture rehabilitation: a 12-month follow-up study. International Journal of Applied and Basic Medical Research, 8(3), 169. https://doi.org/10.4103/ijabmr.IJABMR_171_18

King, T., \& Kapadia, D. (2003). Oral health status and treatment needs of institutionalized elderly and disadvantaged population in Fiji (1997). Pacific health dialog, 10(1), 35-40. Retrieved November 12, 2020, from http://pacifichealthdialog.org.fj/Volume2010/No120Oral20Health20In20The20Pacific/Oral20Health/Origin 
al20Papers/ORALH201B.PDF

Meier, T., Deumelandt, P., Christen, O., Stangl, G. I., Riedel, K., \& Langer, M. (2017). Global burden of sugar-related dental diseases in 168 countries and corresponding health care costs. Journal of dental research, 96(8), 845-854. https://doi.org/10.1177/0022034517708315

Michaud, P. L., de Grandmont, P., Feine, J. S., \& Emami, E. (2012). Measuring patient-based outcomes: is treatment satisfaction associated with oral health-related quality of life?. Journal of dentistry, 40(8), 624-631. https://doi.org/10.1016/j.jdent.2012.04.007

Mushtaq, M., Altaf, J., Sheikh, M., Khan, M., \& Shah, A. (2019). Assessment of Knowledge and Practices about Denture Hygiene among Complete Denture Wearers in Lahore City. Journal of The Pakistan Dental Association, 28(4), 187-191. https://doi.org/10.25301/jpda.284.187

Parvez, K., Parvez, K., Sultan, R., \& Muhammad Aliuddin, A. (2020). Oral Complaints of Complete Denture Wearing Elderly Patients and Their Relation With Age \& Gender. Journal of the Pakistan Dental Association, 29(3), 140-143. https://doi.org/10.25301/JPDA.293.140

Panchevska, S., Elenchevski, S., Janeva, N., \& Mijoska, A. (2018). Satisfaction of complete denture wearers. Medical Review, 71(7-8), 247-249. https://doi.org/10.2298/MPNS1808247P

Pengpid, S., \& Peltzer, K. (2018). The prevalence of edentulism and their related factors in Indonesia, 2014/15. BMC Oral Health, 18(1). https://doi.org/10.1186/s12903-018-0582-7

Perea, C., Suárez-García, M. J., Del Río, J., Torres-Lagares, D., Montero, J., \& Castillo-Oyagüe, R. (2013). Oral health-related quality of life in complete denture wearers depending on their socio-demographic background, prosthetic-related factors and clinical condition. Medicina oral, patologia oral y cirugia bucal, 18(3), e371. https://doi.org/10.4317/medoral.18648

Pournasrollah, A., Negahdari, R., Mahdi, S., \& Bohluli, S. (2016). Evaluation of Gradual Trend of Patients' Satisfaction with Complete Dentures in the Department of Prosthodontics: A Cross-sectional Study. Advances in Bioscience And Clinical Medicine, 4(2). https://doi.org/10.7575/aiac.abcmed.16.04.02.04

Robin, R. P., \& Raj, J. (2015). A Survey of Denture Hygiene in Older Patients. Journal of Medical Science And clinical Research, 7(10), 897-900. https://doi.org/10.18535/jmscr/v3i9.01

Reginato, V. F., Maroli, A., Caldas, R. A., Sánchez-Ayala, A., Spazzin, A. O., \& Bacchi, A. (2017). Relationship between prosthetic factors and oral health-related quality of life in complete denture wearers. Revista Odonto Ciência, 32(2). https://doi.org/10.15448/1980-6523.2017.2.27486

Sanyal, P., Ghanchi, M., Gosavi, S., \& Malik, S. (2013). A Survey of Complete Denture Patients Experiencing Difficulties with their Prostheses. The Journal of Contemporary Dental Practice, 14(3), 524-527. https://doi.org/10.5005/jp-journals-10024-1355

Srisilapanan, P., Korwanich, N., Jienmaneechotchai, S., Dalodom, S., Veerachai, N., Vejvitee, W., \& Roseman, J. (2016). Estimate of impact on the oral health-related quality of life of older Thai people by the provision of dentures through the Royal Project. International journal of dentistry, 2016. https://doi.org/10.1155/2016/1976013

Torres-Sánchez, C., Montoya-Salazar, V., Torres-Lagares, D., Gutierrez-Pérez, J. L., \& Jimenez-Castellanos, E. (2018). Satisfaction in complete denture wearers with and without adhesives: A randomized, crossover, double-blind clinical trial. Journal of clinical and experimental dentistry, 10(6), e585. https://doi.org/10.4317/jced.54871

Tyrovolas, S., Koyanagi, A., Panagiotakos, D. B., Haro, J. M., Kassebaum, N. J., Chrepa, V., \& Kotsakis, G. A. (2016). Population prevalence of edentulism and its association with depression and self-rated health. Scientific reports, 6(1), 1-9. https://doi.org/10.1038/srep37083

Wearers, E. C. D. (2001). Correlation between quality of life and denture satisfaction in elderly complete denture wearers. The International journal of prosthodontics, 14(1), 77-80.

\section{Copyrights}

Copyright for this article is retained by the author(s), with first publication rights granted to the journal.

This is an open-access article distributed under the terms and conditions of the Creative Commons Attribution license (http://creativecommons.org/licenses/by/4.0/). 